\title{
Application of IDL and ENVI Redevelopment in Hyperspectral Image Preprocessing
}

\author{
Long Xue \\ School of Mechanical and Electronical Engineering, East China JiaoTong University, \\ Nanchang 330013, China \\ ultimata@163.com
}

\begin{abstract}
In the last few years, hyperspectral imaging technique has had a bright future for application on nondestructive detection of agricultural products. However, in experiment, there are usually dozens or even hundreds of hyperspectral images. It needs to spend lots of time in preprocessing all the hyperspectral data. Software ENVI is a kind of image processing software, although the users can define their own algorithms and apply to the opened bands in ENVI or the bands and spectra operation of the images, it doesn't provide program to batch process. In this paper, the custom function "normalized" of Interactive Data Language (IDL) is applied to achieve batch processing on multiple hyperspectral images.
\end{abstract}

Keywords: ENVI, redevelopment, hyperspectral image.

\section{Introduction}

Software ENVI (The Environment for Visualizing Images) is image processing software. It is developed by the sciences of remote sensing using Interactive Data Language (IDL). It combines the latest spectral image processing and image analysis technology with an intuitive, user-friendly interface to help you get meaningful information from imagery. It can fast, conveniently, correctly extract information from geography and special image data. Therefore, it has been widely used in science research, environment protection, meteorology, agriculture, mineral and petroleum exploration. Since hyperspectral image combines the character of image and spectrum, so it is applied in agricultural product quality, food safety and nondestructive detection. At home, Hong Tiansheng et al. built prediction model of Chinese pear quality based on hyperspectral imaging technique and artificial neural network [1]. Zhao Jiewen et al. detected the subtle bruises on apple using hyperspectral image [2]. Liu Muhua et al. nondestructively detected soluble solids content and contamination on navel orange surface by hyperspectral laser-induced fluorescence imaging [3-4]. At abroad, M. S. Kim et al. detected fecal contamination on apple, cantaloupes using hyperspectral fluorescence imagery [5-8]. Juan Xing et al. detected surface defects on tomatoes by a hyperspectral imaging system [9]. In experiment, there are usually tens to hundreds of acquired hyperspectral images, and the images need to be preprocessed. Although ENVI software offers interactive computing functions of spectral bands and spectrum, the same preprocessing of hundreds of images takes considerable time and is a very boring job. 
This article uses IDL to write a batch program module based on ENVI, which can achieve the normalization preprocessing of the spectral data.

\section{IDL and ENVI Redevelopment}

\subsection{IDL Introduction}

IDL is the ideal, timesaving solution for data analysis, data visualization, and software application development. The users can quickly and easily use this software to convert the data to images. The converted images can be simple color, full color or threedimensional images. As ENVI software provides the function interface of IDL, so the users can use the built-in IDL functional components, IDL users function, or a custom program. However, these functions must be kept in a directory of ENVI path list to be automatically compiled.

\subsection{Spectral Data Preprocessing}

Because the uneven distribution of light source intensity under the different wave bands and the presence of dark current noise of camera, so the images may contain more noises in some wave bands, therefore, the hyperspectral images must be normalized. Under the same experimental condition, the image of a stand white plate was acquired. The dark image was acquired by completely covering the lens with its cap. The normalization preprocessing is achieved according to equation (1):

$$
I_{\text {norm }}=\frac{I_{\text {sample }}-I_{\text {dark }}}{I_{\text {reference }}-I_{\text {dark }}}
$$

Where: $I_{\text {norm }}$ - the pixel value of the image after normalized preprocessing;

$I_{\text {sample }}$ - the pixel value of a sample image;

$I_{\text {reference }}$ the pixel value of standard white plate;

$I_{\text {dark }} \longrightarrow$ the pixel value of the dark image.

\subsection{ENVI Redevelopment}

The most common method of ENVI software redevelopment is using IDL to develop small application program modules, and call these modules. Meantime, these modules must be stored in the directory "X:|RSIIIDLXX|productslenviXXIsave_add" to be compiled automatically. In this article, the hyperspectral files to be processed were saved in the directory "X:loriginall", and a total of $\mathrm{n}$ hyperspectral files were named according to numerical order (such as DH1.raw, DH2.raw, ..., DHn.raw). In the folder, The standard white plate hyperspectral image (white.raw) and the dark hyperspectral image (black.raw) were saved in the same folder. After preprocessing, the hyperspectral files were stored in the directory "x: $\backslash$ normalized $\backslash$ folder", the program code is as follows: 
; Building the patch processing program module of hyperspectral images "normalized". The function is to normalize the original images.

pro normalized

; Setting the path of hyperspectral files to be processed.

inpath=' $\mathrm{X}: \backslash$ original \'

; Setting the path of hyperspectral files having been processed.

outpath=' X:\normalized \'

; Defining the array "filename" which was used to store the file name of dark image and standard white plate image.

filename $=$ ['black.raw', 'white.raw']

; read the hyperspectral files, L represents the data is numerical type.

for $j=1 L, n L$ do begin

; Converting data from numerical type to character type num=string (format $\left.={ }^{\prime}(i 0) ', j\right)$

; Saving the path and file name of a specific hyperspectral file with "in_name"

in_name0=inpath+num+ '.raw'

; Printing the file name in IDL screen to express the processing course

print, in_name0

; Opening the file, option "no_realize" represents that the file will not be opened in available band list, and "fid" is the number of the file.

envi_open_file, in_name0,/no_realize, r_fid=fid

; Opening the dark and standard white plate images. in_name1=inpath+filename [0]

in_name2=inpath+filename [1]

envi_open_file, in_name1,/no_realize,r_fid=fid2

envi_open_file, in_name2,/no_realize,r_fid=fid3

; Accessing file's information, "ns" represents the sample number of the file, "nl" represents the number of lines, "nb" represent the number of bands, "xstart" and "ystart" represents the pixel 
coordinates of the top left corner in the image and the default value is $(1,1)$.

envi_file_query, fid, interleave=interleave, ns=ns, $\mathrm{nl}=\mathrm{nl}, \mathrm{nb}=\mathrm{nb}, \mathrm{dims}=$ dims, data_type=_datatype, xstart $=$ xstart, ystart=_ystart, SPEC_NAMES=SPEC_NAMES

; "dims" is the spectral range to be processed. "Ssn" represents the first file, and "esn" represents the last file. "sln" represents the first line of the file, and "eln" is the last line.

pos $=1$ indgen $(\mathrm{nb})$

dims $=[-11, \mathrm{ssn}, \mathrm{esn}, \mathrm{sln}, \mathrm{eln}]$

envi_file_query, fid2, interleave=interleave2

envi_file_query, fid3, interleave=interleave3

; Setting the command blocks.

tile_id=envi_init_tile(fid,pos,num_tiles=num_tiles, interleave=interleave $>1, \mathrm{xs}=\operatorname{dims}[1], \mathrm{xe}=\mathrm{dims}[2], \mathrm{ys}=\mathrm{di}$ ms [3], ye=dims [4])

tile_id2=envi_init_tile(fid2,pos,num_tiles=num_tiles, interleave=interleave $>1, \mathrm{xs}=\mathrm{dims}[1], \mathrm{xe}=\mathrm{dims}[2], \mathrm{ys}=\mathrm{di}$ ms [3], ye=dims [4])

tile_id3=envi_init_tile(fid3,pos,num_tiles=num_tiles, interleave=interleave $>1, x s=d i m s[1], x e=d i m s[2], y s=d i$ ms [3], ye=dims [4])

; Processing each block data, and saving the file in the directory " $\mathrm{x}: \backslash$ normalized $\backslash "$

openw, _unit, outpath+num+'.raw', /get_lun

for i=0L, num_tiles-1 do begin

data = envi_get_tile(tile_id, i)

data2 = envi_get_tile(tile_id2, $i)$

data3 = envi_get_tile(tile_id3, i)

data4=(float $($ data $)-f l o a t($ data $)) /($ float $($ data3 $)-$ float (data2))

writeu, _unit, data4

endfor

free_lun, _unit

; Building the head file. 


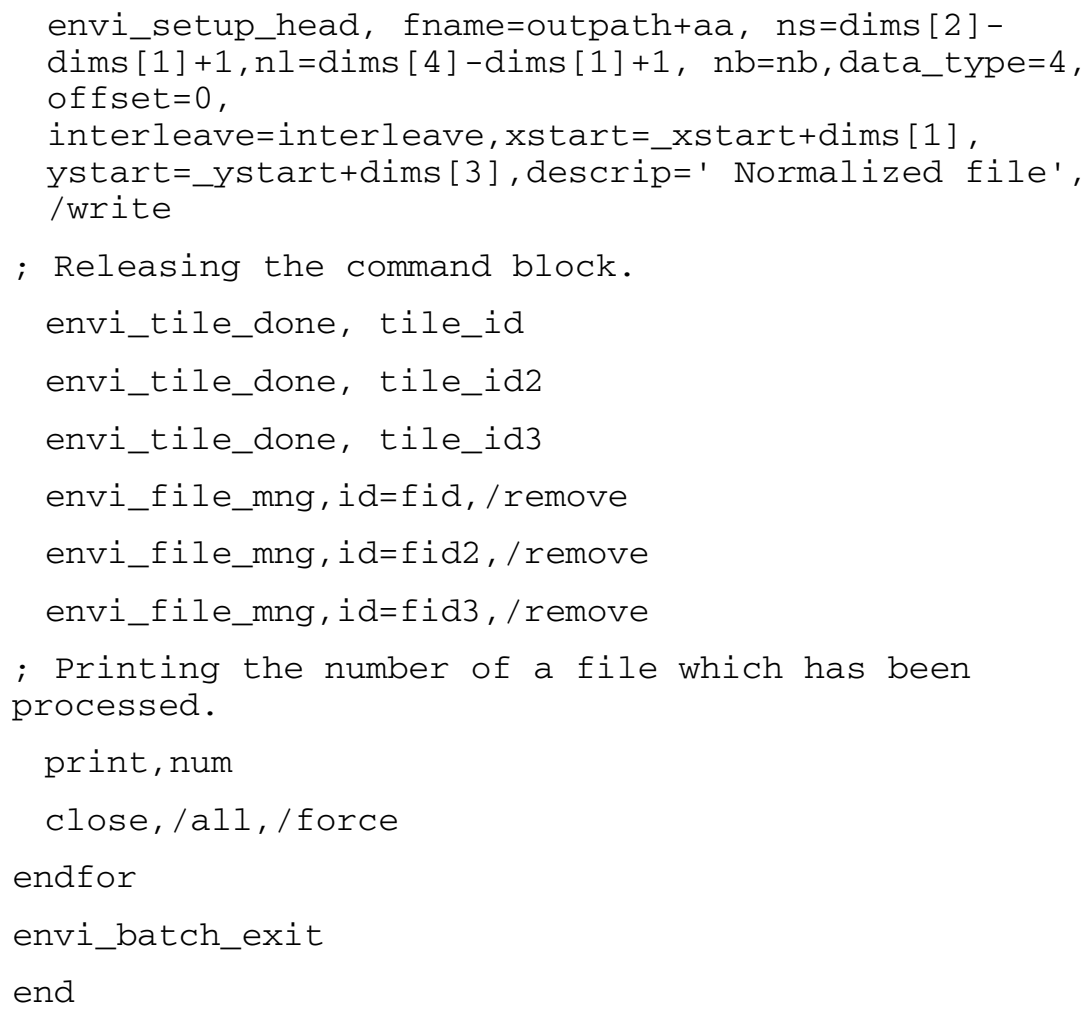

The image of pear at $680 \mathrm{~nm}$ before preprocessing is shown in Figure 1. From figure 1 , the obvious strip in the image is found easily. Figure 2 show the image of pear at $680 \mathrm{~nm}$ after preprocessing, the quality of image is improved greatly.

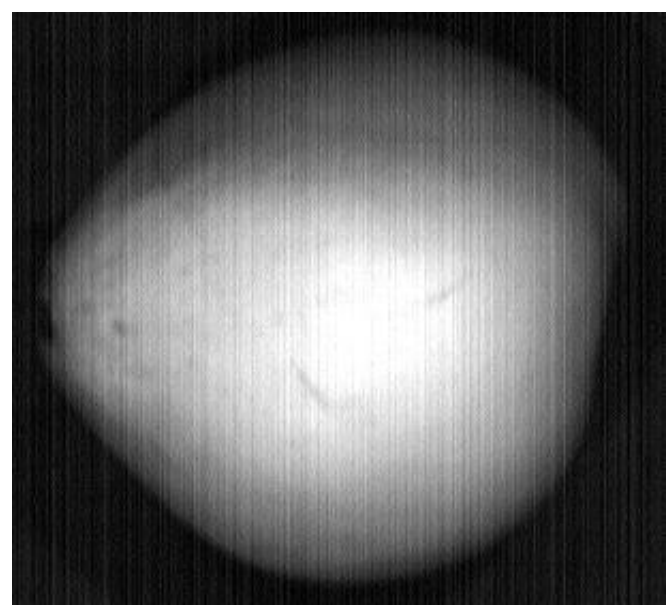

Fig. 1. Image of pear at $680 \mathrm{~nm}$ before preprocessing 


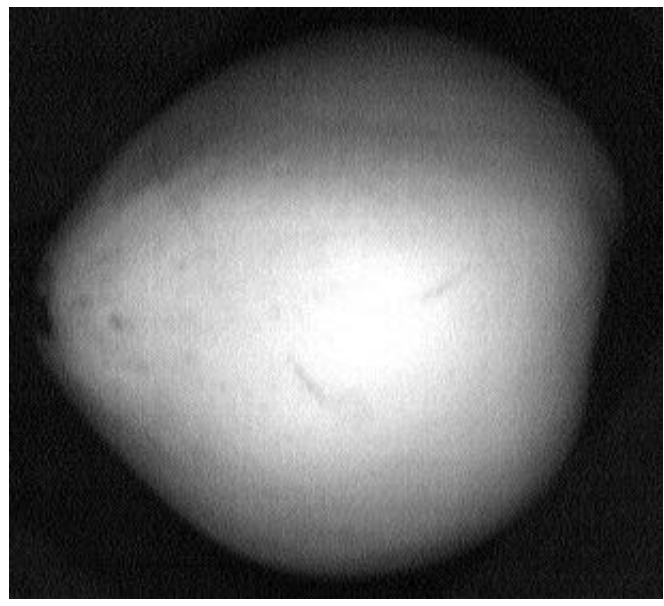

Fig. 2. Image of pear at $680 \mathrm{~nm}$ after preprocessing

Figure 3 show the average visible and near-infrared spectra in the 500 to $950 \mathrm{~nm}$ region of the pear, (a) before preprocessing, (b) after preprocessing.

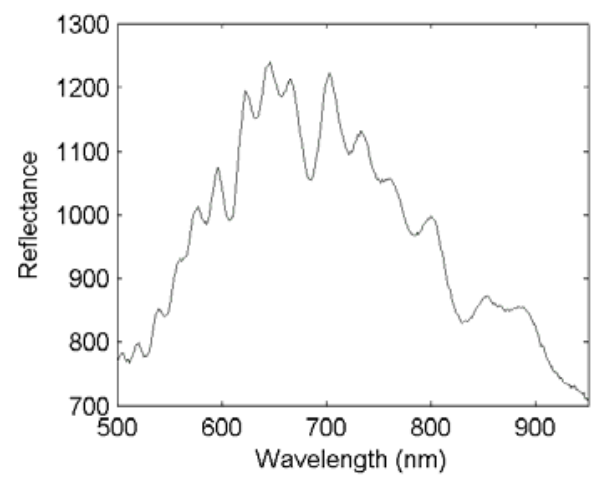

(a) Before preprocessing

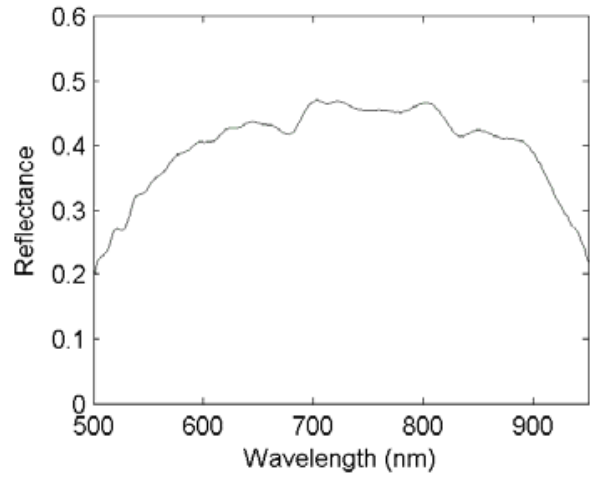

(b) After preprocessing

Fig. 3. The average visible and near-infrared spectra in the 500 to $950 \mathrm{~nm}$ region of the pear 


\section{Conclusion}

By using IDL language and ENVI redevelopment, an extensible remote sensing system with perfect functions can be developed. Nowadays, this system is further expansion because more and more research achievements are integrated in it by scientific researchers. The strong abilities of IDL language and ENV redevelopment make the scientific researchers can release from boring programming work to invest more time and effort to actual research.

\section{References}

[1] Hong, T., Qiao, J., Wang, N., et al.: Non-destructive inspection of Chinese pear quality based on hyperspectral imaging technique. Transactions of the CSAE 23(2), 151-155 (2007)

[2] Zhao, J., Liu, J., Chen, Q., Saritporn, V.: Detecting Subtle Bruises on Fruits with Hyperspectral Imaging. Transactions of the Chinese Society for Agricultural Machinery 1(31), 106-109 (2008)

[3] Guo, E., Liu, M., Zhao, J., Chen, Q.: Nondestructive Detection of Sugar Content on Navel Orange with Hyperspectral Imaging. Transactions of the Chinese Society for Agricultural Machinery 39(05), 91-93, 103 (2008)

[4] Xue, L., Li, J., Liu, M.: Detecting Pesticide Residue on Navel Orange Surface by Using Hyperspectral Imaging. Acta Optica Sinica 28(12), 2277-2280 (2008)

[5] Kim Moon, S., Lefcourt, A.M., Chen, Y.-R.: Ns-scale time-resolved laser induced fluorescence imaging fordetection of fecal contamination on apples. In: SPIE 2004, vol. 5587, pp. 190-197 (2004)

[6] Vargas, A.M., Kim, M.S., Tao, Y., Lefcourt, A., Chen, Y.-R.: Safety Inspection of Cantaloupes and Strawberries Using Multispectral Fluorescence Imaging Techniques. ASAE Paper, No. 043056. St. Joseph. ASAE, Mich. (2006)

[7] Kim, M.S., Lefcourt, A.M., Chen, Y.-R.: Automated detection of fecal contamination of apples based on multispectral fluorescence image fusion. J. Food Engineering 71, 85-91 (2005)

[8] Lee, K-J., Kang, S., Kim, M.S.: Hyperspectral imaging for detecting defect on apples. ASABE Paper No. 053075. St. Joseph. ASABE, Mich. (2005)

[9] Xing, J., Ngadi, M., Wang, N.: Wavelength Selection for Surface Defects Detection on Tomatoes by Means of a Hyperspectral Imaging System. ASAE Paper, No. 063018 St. Joseph. ASAE, Mich. (2006) 